CuPAUAM 25.1, 1998, pp. 9-33

\title{
LA ETNOARQUEOLOGÍA: \\ UNA DISCIPLINA NUEVA EN LA DOCENCIA UNIVERSITARIA Y EN LA INVESTIGACIÓN ESPAÑOLAS
}

\author{
ISABEL RuBIO DE Miguel \\ Profesora Titular de Prehistoria \\ Universidad Autónoma de Madrid
}

\begin{abstract}
Resumen
La Etnoarqueología establece relaciones entre el comportamiento humano y sus restos materiales mediante la observación de grupos actuales que viven al margen de la sociedad industrializada. Se debate aûn su carácter de disciplina independiente o de técnica de trabajo, ya que es de reciente aplicación. La Analogía etnográfica ha sido un recurso común en la investigación del pasado desde siglos atrás. Sin embargo, su desprestigio propicí el nacimiento de la Etnoarqueología que intenta construir modelos explicativos que ayuden a deducciones de carácter arqueológico. Después de reflexionar sobre los objetivos de la misma, su validez y su futuro, se presentan en este arículo diversas experiencias llevadas a cabo en el Dpp de Prehistoria y Arqueología de la U.A.M. y se propone un caso de estudio a nivel teórico destinado a ampliar las posibitidades de interpretación de las relacfones entre cazadores-recolectores y agricultores en la Prehistoria.
\end{abstract}

\section{Summary}

Ethnoarchaeology sets up relationship between human behaviour and its material remains by observing populations who live nowadays out of industrial society. Its identity as independent discipline or as working technique is discussed, because its application is very recent. Erhnografical Analogy has been an ordinary implement in research since past centuries. But its lost of credit favoured Ethnoarchaeology emergence trying to build expljcative models which would help to came to archaeological conclusions. After thinking over Ethnoarchneology aims, its validity and future, some experiences made in the Prehistory and Archaeology Department of the U.A.M. are explained in this paper and a study case is proposed at a theoretical level in order to enlarge understanding posibilities about relationship between hunter-gatherers and farmers in Prehistory.

La Etnoarqueología podría ser definida como la disciplina que se ocupa de establecer las relaciones entre el comportamiento humano y sus residuos tangibles mediante la observación de grupos actuales que viven al margen de la sociedad industrializada. 
Sin embargo, inmediatamente cabría efectuar ciertas precisiones y recordar que, precisamente, aún se debate su propio carácter: ¿disciplina independiente o procedimiento de estudio?. La conciencia de su incierto futuro y el deseo de ampliar horizontes por otra parte ha hecho que se dirija tambiên a otro tipo de sociedades. Recordemos a este respecto el Garbage Project llevado a cabo en su momento en la ciudad de Tucson (Arizona)(Rathje, 1978), pero también estudios más o menos recientes realizados en nuestro país (Cerrillo, 1989 o Molinos et al., 1996, por ejemplo).

Aứn teniendo en cuenta que la identidad misma de la Etnoarqueología se discute, ha sido integrada en los planes de estudio de algunas universidades españolas, la U.A.M. entre ellas, dentro del itinerario de Prehistoria y Arqueología de la Licencianura de Historia. Cada vez son más numerosos también los trabajos de investigación llevados a cabo con este enfoque, por lo que ya no resulta extraño tropezamos con hipótesis basadas en documentacion etnográfica de diverso tipo. Sin embargo, la necesidad que ha conducido a la inclusion de esta materia como algo independiente en los planes de estudio que afectan al conocimiento del pasado únicamente tiene su razón de ser en las escuelas europeas, puesto que en las americanas se halla implícito en la Antropología cultural desde la que se aborda la investigación arqueológica, por lo que en éstas y en otras se asume como una forma normalizada de acercamiento al pasado.

Dos han sido los artículos pioneros en nuestro país sobre la Etnoarqueología y sus peculiaridades. Uno de ellos, publicado por V Fernández (1994), supuso un primer acercamiento en castellano y en España a los métodos y aplicaciones de la misma, tal como indicaba su título, y el segundo, aparecido un año después y redactado por A. Hernando (1995), abordaba fundamentalmente su definición y objetivos, además de un caso de estudio relacionado con las sociedades agricolas (con el Neolítico en el caso de la Prehistoria). La referencia a ambos articulos, de obligada consulta para cualquier persona interesada en el tema, me excusa el profundizar en las cuestiones concretas que en ellos se exponen.

El establecimiento de paralelos etnográficos se produce desde el siglo pasado por parte de los prehistoriadores europeos. Los americanos se enfrentaban desde el inicio de la investigación con las complejas sociedades de indios de ese continente. No obstante, como tal disciplina no aparece hasta hace 20 \% 25 años, siendo además los propios arqueólogos los que llevan adelante esta tarea (Renfrew y Bahn, 1993, 61).

¿Qué es realmente la Etnoarqueología?. Algunas de las definiciones aportadas por diversos investigadores permiten comprobar que coexisten enfoques diversos bajo este mismo término. $O$, a la inversa, que se aplican distintas denominaciones a algo que, en general, se acepta como planteamiento etnoarqueologico, to sea o no: Arqueología en acción, Arqueologia viva, Arqueoetnografía y Arqueología etnografica (Kramer, 1979, 12).

Desde la definición aportada por R. Asher en 1961 que hacía referencia a la formación del registro arqueológico y a la verificación de hipotesis reconstructivas, fundamentalmente, o la acuñada por M.B. Stanislawski en 1974, que se dirigía más bien al estudio de la fabricación, distribución y uso de los útiles en su contexto "a fin de construir mejores modelos explicativos que sirvan de ayuda a deducciones de carácter arqueológico"(Guidi, 
1988, 220), encontramos otras proporcionadas por investigadores estrechamente ligados a los inicios de la Etnoarqueología.

C. Kramer $(1979,1)$ consideraba en su momento que "Ethnoarchaeological research investigates aspects of contemporary sociocultural behaviour from an archaeological perspective; ethnoarchaeologists attempt to systematically define relationship between behaviour and material culture not often explored by ethnologists, and to ascertain how certain features of observable behaviour may be reflected in remains which archaeologists may find"

R. Gould, por su parte, prefería denominarla Arqueología viva: el esfuerzo realizado por arqueólogos y etnografos para llevar a cabo trabajo de campo en sociedades vivientes con especial referencia al patrón "arqueológico" del comportamiento de estas sociedades (Vossen, 1990, 24). Del mismo modo, Vossen (1990, 24) señalaba, más recientemente que Arqueología viva es "el tratamiento arqueologico de objetos documentados etnográfica* mente, asî como el trabajo de campo etnográfico con una formulación arqueológica de la cuestión"

Por último, I. Hodder (1988, 128-129) consideraba desde la corriente post-procesual que la Emoarqueología pasa a ser el estudio de la Arqueología en contextos etnográficos con el fin de arrojar luz sobre el presente etnográfico, con lo que se alinearía con la Etnohistoria, tanto por lo que se refiere a su definición como a su práctica.

Como es fácil observar, las definiciones de Etnoarqueología comprenden desde planteamientos muy concretos a intentos de generalización diversos, por una parte, y por otra, la contemplan desde diferentes ángulos. Sin embargo, conviene examinar cómo han llegado a generarse todos éstos y otros que veremos para comprender adecuadamente esta diversidad.

\section{UN POCO DE HISTORIA}

Las raíces de esta forma de acercamiento al pasado ( $y$ al presente) pueden rastrearse mucho más atrás. Sin embargo, el enfoque ha variado a través del tiempo. El descubrimiento de América en 1492 y la posterior vuelta al mundo proporcionaron numerosos datos sobre las nuevas poblaciones halladas que fueron dados a conocer a través de los escritos de los cronistas de Indias o de las Relaciones de los jesuitas.

Los siglos XVI y XVII marcan el inicio de la exploración y la colonización de todo el mundo por los países de la Europa occidental. Los marinos se familiarizan con los gupos de cazadores-recolectores y de agricultores de América, África y el Pacífico. Comienzan a circular descripciones de estos pueblos y sus costumbres, ropas y utensilios se muestran como una curiosidad. De ese modo, se empieza a esbozar una visión alternativa de nuestros antepasados más remotos, basándose en las poblaciones citadas.

En el siglo XVII, las descripciones de los exploradores y las ilustraciones de los dibujantes que les acompañaban, además de las noticias de los misioneros, proporcionaron el 
conocimiento del modo de vida de pueblos ignorados hasta entonces. Se publican obras como la del jesuita Lafitau, por ejemplo: Moeurs des savages americains comparée aux moeurs des premiers temps, de 1724, que establecia el paralelismo entre los indigenas americanos y los representantes de los primeros tiempos de la Humanidad. El siglo XVIII, en todo caso, disponía de una visión global del mundo y de otras sociedades distintas de las europeas cuyos modos de vida se asociaron a los momentos más antiguos de la vida del hombre, acorde con la noción de evolución y progreso que se halla entre los pensadores de la Ilustración. En todo caso, estos grupos eran considerados una degeneración de la civilización cristiana.

En el siglo XIX, según el paradigma evolucionista, la historia era vista como un único proceso de desarrollo evolutivo, por lo que los arqueólogos y etnógrafos se concentraron en los restos correspondientes a su campo, es decir, los tiempos prehistóricos en è caso de los primeros y la conducta de comunidades existentes fuera de las fronteras del mundo industrial en el de los segundos. El comparativismo, muy próximo al actualismo de la Geología expuesto por Ch. Lyell, hizo que se considerara lícito que los unos tomaran datos de los otros y los extrapolaran.

Se establece, por otra parte, una jerarquización de las sociedades humanas que trae como consecuencia la denominación de los primitivos contemporáneos como sociedades sin historia, lo que demuestra la ignorancia de los hechos concretos (Mercier, 1995, 59). En este mismo siglo, nacen en las escuelas europeas la Prehistoria y la Etnografia, de la que rápidamente se desgajará la Etnología, como disciplinas independientes.

En 1854, F. Keller estudia los restos palafíticos que paraleliza con los hăbitats existentes hasta hoy en zonas tropicales (Guidi, 1988, 16) (recientemente los Petrequin han llevado a cabo un estudio etnoarqueológico en Nueva Guinea destinado a interpretar los restos de los poblados lacustres alpinos) (Petrequin y Petrequin, 1988).

La Analogía etnográfica se convierte entonces en una forma común de acercamiento al pasado. Así, J. Lubbock, en su obra Prehistoric times publicada en 1865, comparaba asentamientos arqueológicos con los modos de vida de bosquimanos o esquimales (Guidi, 1988, 19) y sugería que los instrumentos líticos de estos últimos eran muy similares a los del Paleolítico superior europeo. Asimismo, E. Cartailhac securría a la cultura tasmania para interpretar la vida de los cazadores-recolectores prehistoricos (Almagro, 1967, 123125).

Sin embargo, serán los estudiosos que trabajan en los Estados Unidos los primeros en poner de manifiesto la incorrección que suponía relacionar las tribus de Norteamérica con pueblos de otras zonas del mundo. Por ese motivo, $\mathrm{F}$. Boas señala la conveniencia de interpretar los elementos culturales de la Arqueología a través de la Etnología/Antropología de la misma región y no de áreas más amplias (Laurencich-Minelli, 1966, 67-68).

En los inicios del siglo XX, la Analogía etnográfica se emplea comunmente, incluso de forma mecánica, considerando a pueblos contemporáneos como los sucesores directos de determinadas culturas paleolíticas (Guidi, 1988, 51 y Fernández, 1994, 140). Sin embargo, la aplicación de un verdadero método etnoarqueológico se produce ya en 1921 con O.G.S. Crawford. En Man and his past, señalaba cómo en una visita a un campamento de 
Sudán, abandonado después de un incendio, se dio cuenta de que la distribución de los objetos sobre el terreno era en algún caso similar a la del yacimiento que excavaba en ese momento (Guidi, 1988, 77), lo que contribuyó a la interpretación de este último.

Otros investigadores adoptaron planteamientos similares. W.D. Buttler, por ejemplo, permaneció durante dos meses con los campesinos húngaros, rumanos y yugoslavos antes de redactar la memoria definitiva del yacimiento de la cultura de la cerámica de bandas de Köln-Lidenthal. Investigadores escandinavos desarrollaban paralelamente estudios de lo que hoy denominaríamos Arqueología experimental, tan ligada a la Etnoarqueología (Guidi, 1988, 77). En 1939, D.F. Thomson estudiaba el grupo australiano de los Wik Munkan y demostraba que las actividades anuales implicaban desplazamientos estacionales que daban como resultado una distribución espacial de yacimientos distintos entre sí (los arqueólogos tradicionales los habrían atribuido a culturas distintas) (Guidi, 1988, 143).

Con todo, en los años sesenta, el método etnológico (o la Analogía etnográfica) se hallaba aún en vigor en ciertos ámbitos. Pero, de la misma manera, había sufrido un considerable desprestigio a causa de las limitaciones observadas: los objetos han cambiado su función y paralelismos como los establecidos conducían a graves errores de interpretación.

Con la aparición de la New Archaeology americana se produce la irrupción de la Antropología cultural en los estudios del pasado (Binford, 1962, por ejemplo). Paralelamente, la carencia de publicaciones etnográficas orientadas a la cultura material lleva a los arqué́logos a considerar la necesidad de aplicar los procedimientos que caracterizan a la moderna Etnoarqueología. La idea inicial de la Nueva Arqueología era la superación de los problemas del método analógico, pensando que los datos de carácter etnográfico eran la fuente de hipótesis de comportamiento que después se comprobaban cien. tíficamente con los datos arqueol6gicos generando otras hipotesis (Fernández, 1994, 160. 163). V. Fernández $(1994,141)$ señala cómo la corriente procesual ha aspirado, además, a generalizaciones teóricas sobre las relaciones entre comportamientos y cultura material que trascendieran el contexto histórico de una región o cultura determinada (abandonando la aproximación histórica directa) y que sirvieran, bajo ciertas condiciones, para el trabajo arqueológico generalmente considerado (leyes de cultura comparada).

L. Binford (1988, 27-28), que puede considerarse el principal impulsor de la Etnoarqueología, sostiene que, si intentamos investigar la relación existente entre lo estático y lo dinámico de una cultura, deberíamos de poder observar ambos aspectos simultáneamente y el único lugar donde podemos hacerlo es en el mundo actual. Así, por ejemplo, uno de sus trabajos en el desierto de Australia se basaba en la observación de grupos que fabricaban objetos líticos (lo estático) con objeto de correlacionarlos con su comportamiento (lo dinámico). Para él sería necesaria una observación objetiva, desde fuera, no participante, capaz de desarrollar relaciones entre estos dos niveles, lo que podría lograrse mediante la Teoría de Alcance Medio: instrumentos de medición independientes para poder "leer" los datos arqueológicos y desarrollar argumentaciones de cierta relevancia acerca de las relaciones entre la cultura material y la sociedad (Hodder, 1988, 127).

A comienzos de los años sesenta, el enfoque etnoarqueológico varió un tanto en Estados Unidos. Si en un principio se había aplicado a los tradicionalmente denominados 
pueblos primitivos actuales, desde ese momento se fue ampliando a la sociedad industrial en la idea de que se puede estudiar ésta desde el punto de vista arqueológico. En Europa, la Etnoarqueología entendida como disciplina independiente con una metodología clara y diferenciada, asi como con propósitos igualmente distintos, no se encuentra hasta la década de los noventa del pasado siglo XX. Pero no todos los estudios denominados etnoarqueológicos lo son realmente y, en ocasiones, es posible hallar trabajos de carácter etnohistórico, por ejemplo, bajo el anterior calificativo. Otros investigadores han adoptado una postura crítica frente al trabajo ya realizado.

Ian Hodder (1978 y 1982), representante de la Arqueología contextual, por ejemplo, no está de acuerdo con los planteamientos de Binford o de Gould. Sus investigaciones entre los Baringo de Kenya han tratado de demostrar que es necesario sumergirse en la información contextual (Guidi, 1988, 231 y 233-234). En su opinión, los métodos de la Etnoarqueología déberían implicar el estudio desde el interior, la participación y el análisis histórico (Hodder, 1988, 130-143).

Por último, cabría preguntarse ¿qué no es Etnoarqueologia?. A. Hernando (1995) ha establecido claramente sus diferencias con otras disciplinas o procedimientos que parecen confundirse con ella. Así por ejemplo, precisa con respecto a la varias veces citada Analogía etnográfica que ésta establece semejanzas entre casos particulares y no diferencias, mientras que la Etnoarqueología tratan de establecer generalizaciones que aspiran a tener el rango de leyes: llegar a comprender bajo qué circunstancias se puede esperar un cierto tipo de comportamiento o la aparición de un cierto documento material. Del mismo modo, la Arqueología viva, cuyo objeto de estudio sería la cultura material de los pueblos vivos desde una perspectiva arqueologica, difiere de la Etnoarqueología en que esta segunda compara pautas etnográficas y arqueológicas, por lo que, en todo caso, englobaría a la primera.

Este repaso, forzosamente breve, de la historia de la Etnoarqueología puede explicar con claridad los motivos de su nacimiento, sus verdaderos objetivos y también sus diferencias con enfoques similares. Sin embargo, seria preciso hacer referencia igualmente a otros aspectos de la misma.

\section{ALGUNAS PARTICULARIDADES DE LA ETNOARQUEOLOGIA}

Sus objetivos son en sentido estricto aquellos senalados por L. Binford, vistos anteriormente. Pero, existen otras razones concretas para acometer una investigación de estas características? En las sociedades ágrafas de la Prehistoria, la información sobre los aspectos no tangibles de la cultura es mucho más dificil de interpretar a través de los restos materiales. V. Fernández $(1994,138)$ opina que, de hecho, toda interpretación arqueológica de los restos del pasado y no sólo, se basa en observaciones y teorías actuales por la imposibilidad de un acceso directo, lo que se produce igualmente en Paleontología, Geología, etc. A partir de la irrupción antes señalada de la Antropología cultural ("la Arqueología es Antropología o no es nada", afirmación que puede ser debatida, en todo caso), la atribución de las características de los grupos prehistóricos, fundamentalmente de cazadores- 
recolectores se ha hecho desde la analogía con poblaciones vivas, comenzando por las categorías de sociedades establecidas por los antropólogos (Fried, 1967 y Service, 1971, fundamentalmente). Nadie ha visto un inconveniente en ello, habida cuenta que, a determinados tipos de actividad parecen corresponderse, generalmente hablando, ciertas clases de organización social.

Estas mismas razones han propiciado que una buena parte de esta disciplina se haya centrado en el estudio de los cazadores-recolectores, por lo que se constata una preferencia por poblaciones de Alaska, África y Australasia. Por otra parte, este hecho se justifica también porque la mayor parte de la Humanidad lo fue y porque urge su estudio antes de su desaparición o de su integración en grupos más complejos (Kramer, 1979, 3). La costumbre ha sido considerarlos aislados y no articulados con otros semejantes o más complejos. J. Mercader (1993) ha insistido en que la suposición de primitivismo no sería la más adecuada para designar a gentes que viven también en el presente, desde el cual ha de interpretarse el pasado. Los muchos estilos de vida diferentes que presentan, fruto de adaptaciones distintas, nos brindan la oportunidad de reflexionar acerca de las causas de su diversidad y cómo se podrían investigar.

Sin embargo, tambien se han llevado a cabo estudios en sociedades más complejas: de pescadores, pastores nómadas, agricultores e incluso industriales.

Lo reciente de su aplicación y también lo fragmentario de la misma se hallan en el origen del debate planteado acerca de su entidad. En opinión de Kramer (1979, 1), la Etnoarqueología surge como un enfoque útil en el estudio de los restos arqueológicos. Se asume que ciertos elementos comportamentales tienen su correlato material. Si éstos se incorporan al documento arqueológico, pueden servir para desarrollar inferencias sobre el comportamiento al que están asociados. La observación del comportamiento actual puede ser útil sobre todo si existen similitudes en el entorno y en la tecnologia.

Vossen $(1990,29)$, en cambio, cree que se trata de una subdisciplina de la Antropología, que se define en sentido amplio por acompasar todos los aspectos teóricos y metodológicos al comparar los datos etnográficos y arqueológicos, incluyendo el uso de la Analogía etnográfica y de la Etnografía arqueológica.

Así pues, por un lado, se tiende a considerarla como una disciplina independiente con identidad definida, integrada en planes de estudio universitarios en nuestro caso y, en otros, se perfila como una parte de materias más amplias o simplemente como una técnica de trabajo.

Posiblemente, una breve referencia a la metodología etnoarqueologica contribuya a clarificar también algunos de estos aspectos. Se asume que la puesta en marcha de una investigación etnoarqueológica parte de un problema arqueológico. Para su resolución, dicha metodología implica que el investigador, al desarrollar el trabajo de campo, se traslade a vivir con una determinada comunidad contemporánea cuyo modo de vida sea similar a una histórica o prehistórica que se trata de comprender y que habitualmente ha sido estudiada por etnólogos. La duración de la estancia dependerá de los aspectos que se proponga investigar. El etnoarqueólogo observa y estudia cómo se produce el documento arqueologico en esa sociedad viva. En ocasiones, se realizan también excavaciones en 
poblados que han sido abandonados por estos grupos. A veces, el trabajo de campo es más limitado y se reduce a visitas y entrevistas relacionadas con los temas que se van a estudiar. Igualmente, puede que no se lleve a cabo un trabajo de campo directo si se conocen datos suficientes ya publicados sobre el aspecto en cuestión. Los datos obtenidos mediante la entrevista y la observación, participante o no, se integran con los publicados de carácter etnográfico, recopilándolos para evaluar hipótesis que, inicialmente, derivan de interpretaciones de datos arqueologicos y de observaciones etnográficas independientes.

\section{LA VALIDEZ DEL PROCEDIMIENTO ETNOARQUEOLÓGICO: POSIBHIDADES Y LMM- TACIONES}

Podrian hacerse también algunas reflexiones acerca de la validez de estos estudios y sobre las condiciones que deben reunir para ser considerados fiables en algún grado, sobre todo, cuando se produce su extensión a cualquier tiempo y lugar.

De nuevo, V. Fernández $(1994,138)$ señala que para que tal aplicación sea vâlida hay que suponer un nexo entre el hombre y el objeto que haya permanecido invariable a lo largo de los años. Por esta razon, las primeras investigaciones se llevaron a cabo en regiones en las que el poblamiento era continuado (aproximación histórica directa), como Norteamérica o Australia. En el mismo sentido, se habían pronunciado, como ya hemos visto, investigadores que trabajaban en el continente americano. Pero también, más recientemente, $O$. Aurenche (1995) consideraba la continuidad historica como una de las tres condiciones que, en su opinión, debería cumplir la investigación etnoarqueológica. La situacion ideal sería el estudio de una sociedad con descendientes directos que, mediante la encuesta oral, pudieran clarificar los restos materiales. Sin embargo, este mismo autor, tratando de facilitar la aplicación de la Etnoarqueología en áreas donde este requisito no se cumple, propone tratar sobre civilizaciones pastoriles, agro-pastoriles o urbanas, por ejemplo, e interrogarse sobre la persistencia de sus rasgos culturales a través del tiempo, en lugar de hablar de civilizaciones desarrolladas desde los inicios de la Historia (Aurenche, 1995, 13-14)

Las otras dos condiciones restantes serían la continuidad geográfica y el contexto socio-económico. Para la primera de ellas se propone la comparación entre ecosistemas de costa, de media o alta montaña, de desierto o de estepa. El contexto geográfico podría tratarse de dos maneras: comparando sociedades alejadas en el tiempo pero implantadas en la misma zona geográfica o comparando sociedades alejadas en el espacio y en el tiempo implantadas en microambientes similares (Aurenche, 1995, 14-15). Por último, se trata de poner de manifiesto la presencia o ausencia de ciertas técnicas en el contexto de las sociedades estudiadas. Las técnicas, que por regla general han constituido una vía eficaz de acercamiento al pasado, no coinciden forzosamente en su aparición con el momento que se les habia atribuido inicialmente. Con todo, $O$. Aurenche $(1995,15-16)$ indica que, al darse innumerables situaciones diferentes, se haría preciso introducir filtros para situar la documentación etnográfica en el mismo grado de generalidad que la arqueológica y evitar así caer en una Etnoarqueología "puntillista", lo que, en mi opinión, está sucediendo.

Lo cierto es que las conclusiones se tratan de aplicar de forma general, bien es verdad que desigualmente. Las causas de esta generalización son, en opinión de V. Fernández 
(1994, 138), fundamentalmente tres: la certeza de que el comportamiento del Homo sapiens presenta algunas regularidades que trascienden los límites geográficos y temporales; las teorías evolucionistas que presentan fases culturales similares en el tránsito histórico de la Humanidad y la unidad de "reglas. "interpretativas por parte de los arqueólogos.

En otro orden de cosas, a las limitaciones que se desprenden de todo lo dicho hasta aquí, se unen otras de difícil resolución. C. Kramer $(1979,2)$ indicaba ya, en su momento, cuáles podían ser, a su juicio, las principales dificultades a la hora de plantear un estudio etnoarqueológico. Por un lado, no todos los comportamientos del pasado tienen análogos disponibles hoy para la observación. A la inversa, no podemos asumir que todas las formas de comportamiento cultural que se pueden observar en la actualidad tengan analogías en el pasado. Un ejemplo lo constituirían los humanos anteriores al Homo sapiens, pero tampoco en el caso de éste se hallarían siempre analogías en el momento presente.

\section{EL FUTURO DE LA ETNOARQUEOLOGIA}

L. Binford (1988) recordaba que el documento arqueológico está con nosotros. No se trata de observaciones directas del pasado, sino que forman parte de nuestro mundo contemporáneo. La cuestión es ¿cómo acceder al pasado desde el presente?. Según sus planteamientos vistos al inicio, la solución radicaría en el estudio de pueblos actuales. En su opinión, ésta no se hallaría excavando más, ya que no se puede excavar un yacimiento correctamente sin conocer el potencial que pueden tener sus datos para lograr inferencias sobre el pasado. Tres campos a desarrollar para la consecución de estos fines, siempre en opinión de Binford, serían la Arqueología experimental (se usa el presente para servir al pasado), el examen de los documentos históricos (describen los hechos de hombres del pasado) y la fotografia que constituye otra fuente de información desde el momento de su aparición. La Arqueología, en fin, debía encontrar un equilibrio entre los intereses teóricos y los prácticos.

La Mesa Redonda celebrada en Madrid, en la Casa de Velázquez (Bazzana y Delaigue, 1995) suponía la elección de un momento en el que, según los organizadores, se producía un hito en la práctica etnoarqueológica: un doble movimiento que se traducía en un auge de la misma pero contradictoriamente también en un deterioro. El coloquio se pro ponía una dirección también doble: ofrecer un balance que tuviera en cuenta las prácticas metodologicas y los presupuestos teóricos (elaborados a posteriori) y ampliar el campo de aplicación de la Emoarqueología a los investigadores que trabajan en periodos históricos o a etnólogos interesados en la cultura material (Bazzana y Delaigue, 1995, 7-11).

Si la Etnoarqueología se ha venido aplicando a sociedades ágrafas, esto es, aquellas que ofrecian mayores similitudes con las prehistóricas por razones obvias, no es menos cierto que el campo de trabajo se va reduciendo de una manera drástica por varias razones. La toma de contacto de muchos de los grupos tradicionalmente denominados primitivos actuales con la sociedad industrializada que, aunque no los integra plenamente, desvirtúa sus costumbres y su equipo material, sería una de ellas. Otra, la mâs dramática, sería la extinción por diversas causas de algunas de estas poblaciones. 
Ante esta situación, algunos autores proponen tareas concretas para el futuro. Vossen (1990, 23-41), por ejemplo, señala tres fundamentales. La Antropología y la Arqueología deben recoger datos de interés para la interpretación de testimonios arqueologicos, realizando trabajo de campo etnoarqueológico en distintas partes del mundo donde la historia - el presente etnográfico presenta grados en Arqueología. Ambas disciplinas deberán estudiar, revisar y recoger la bibliografia etnográfica importante de forma sistemática y crítica para realizar un banco de datos etnoarqueológico. Finalmente, será preciso desarrollar un sistema operativo o una teoría "sensata" para rellenar el espacio entre hallazgos arqueologicos y analogias etnográficas de forma sistemática.

\section{ALGUNAS EXPERIENCIAS EN EL DEPARTAMENTO DE PREHISTORIA Y ARQUEO- LOGIA DE LA U.A.M.}

Todo aquel que haya abordado la tarea de preparar un programa docente de Etnoarqueología, como ha sido mi casoill experimentará la repercusión de la desigualdad de datos existente, tanto cualitativa como cuantitativamente, a la hora de equilibrar el estudio de las diversas sociedades. Ello demuestra que buena parte de los interrogantes planteados o por plantear no ha sido resuelta. Por otra parte, resulta verdaderamente compleja la generalización necesaria desde el punto de vista pedagógico. De ahí la dificultad en conseguir un programa no "puntillista", parafraseando a $O$. Aurenche. Sin embargo, pese a las dificultades, ha sido posible desarrollar además, individualmente o en colaboración, algunas actividades interdisciplinares relacionadas con la Etnoarqueología, tratando de aunar la labor docente, teórica y práctica, con el trabajo de campo y la investigación.

Una primera colaboración entre investigadores y docentes procedentes de distintas áreas de conocimiento se produjo con el trabajo de campo etnográfico realizado en diversos términos municipales de Madrid como consecuencia de la elaboración de la Carta Arqueológica de dicha Comunidadi2i. Los resultados fueron publicados en su momento (González, Rubio y Valiente, 1995) y, a pesar de tratarse de una investigación llevada a cabo para estudiar distintas construcciones auxiliares que se hallaban en vías de desaparición (chozos de pastor, fundamentalmente), es decir de carácter puramente etnográfico, el enfoque aplicado contemplaba procedimientos empleados igualmente en los estudios arqueológicos como pudieran ser criterios de Arqueología espacial.

Personalmente y a posteriori, considero que dicho trabajo se reveló también como fuente de conocimiento para sociedades pastoriles en general, ya que quedaba patente que

[1] Dicha asignatura es una optativa de segundo ciclo del itinerario de Prehistoria y Arqueologia del plan de estudios de Historia de la U.A.M.

[2] Ia Dra. Consolación Gonzălez del Museo de Artes y Tzadiciones Populares de la U.A.M., el Dr. Santiago Valiente de la Escuela Superior de Conservacion y Restauración de Bienes Culmurales de Madrid y yo misma llevamos a cabo este imbajo en los términos de Valdilecha, Campo Real, Villas del Oimo, Olmeda de las Fuentes, Corpa y Nuevo Baztán. 
la destrucción de dichos chozos suponía la pérdida completa de la noción de su existencia. Ello era debido a la ausencia de cimientos, tanto para el chozo como para el corral, a la reutilización de la piedra en que se hallaban construidos en otras edificaciones y a la presencia de amontonamientos de la misma ("majanos"), procedentes de la limpieza de los campos de cultivo. Unicamente, la presencia del talón (piedra clave de la cubierta realizada por aproximación de hiladas) revelaba que tales amontonamientos habían sido chozos en su momento. Una excavación llevada a cabo en Brieva de Cameros que, en este caso, se planteó para verificar la cronología de "círculos de piedra" de la zona que resultaron ser históricos, muestra problemas similares (Muntión y Vegas, 1991). En nuestro caso, la transformación de los terrenos de pasto en zonas de labor para viñas y olivos suponía también la pérdida de documentación sobre una primitiva actividad de gran importancia en dichos términos: la ganadera. Por último, la entrevista personal nos proporcionó los datos necesarios sobre la construcción de los chozos y también sobre la organización del sistema ganadero a que respondían, que podía variar incluso de un término a otro, distinto de la trashumancia tradicional de mayor envergadura, a pesar de compartir algunas de las vías de tránsito. Del mismo modo, tuvimos conocimiento de las costumbres y rituales ligados al mismo. Evidentemente, la documentación histórica contribuyó a un mejor conocimiento de esta problemática.

Se ponía así de manifiesto la importancia de la recogida de todos estos datos, ya que únicamente con la metodología arqueológica hubiera sido imposible en el futuro, no ya una interpretación correcta de las actividades económicas del área, sino la constatación de su propia existencia. De nuevo, se evidenciaba la especificidad de las dificultades planteadas por el estudio de grupos que practican un pastoreo con desplazamientos de diverso tipo a través de sus restos materiales. Es éste de un tema que preocupa a los etnoarqueólogos (Aurenche, 1984 y Cribb, 1991, entre otros), precisamente por la escasez o la indefinición de dichos restos desde el punto de vista arqueológico y por la fragilidad de los mismos, debida a las materias primas empleadas. Éstas se hallan condicionadas por la ligereza necesaria en un equipo material destinado a sufrir frecuentes traslados motivados por la alta movilidad de los pastores.

Con posterioridad, se llevaron a cabo una serie de exposiciones en la U.A.M. que mostraban los resultados de las prácticas realizadas con alumnos de diversas materias, siendo coordinadas por los profesores de las mismas con objeto de ofrecer una visión etnoarqueológica de distintos aspectos de la Prehistoria ${ }^{[3]}$. Las exposiciones celebradas hasta la fecha han sido cincolid, sobre temas tan diversos como: "El trabajo agricola y el adorno en las sociedades neolíticas peninsulares" (mayo de 1994); "La tecnología cerä́mica"

[3] La Dra. Consolación Gonźslez del Museo anteriornente citado coordinó la documentación etnográfica, el Dr. Javier Baena la procedente de determinados momentos de la Prehistoria y de la Arqueología experimental y yo nisma la de otras etapas prehistoricas y la obtenida por la Etnoarqueología. Ocasionalmente, colaboraron otros miembros del Departamento como las Dras. Lucas, Gutierrez y Liesau.

[4] Personalmente, he colaborado también en otras sobre "Mascaradas de invierno", organizada por el Museo ya indicado en 1996 y sobre "Materia, forma y simbolo: los origenes", organizada por el Dpt de Prehistoria y Arqueología de la U.A.M. en 1999. 
(abril de 1995); "La caza: instrumentos y estrategias" (mayo de 1995), que además se exhibió en dos centros relacionados con fauna local de la provincia de Córdoba (el parque natural de la laguna de Zóñar y el de Hornachuelos); "Los instrumentos musicales en la Prehistoria y en las sociedades tradicionales actuales" (mayo de 1997), que igualmente se mostró fuera de la U.A.M. (Aranda de Duero, Burgos, en 1997) y "El uso de las fibras vegetales en las sociedades prehistóricas y tradicionales" (mayo de 1998) (VV.AA., 1995, 1995-1996, 1997 y 1998). En algunas de ellas, se estableció la colaboración con personas e instituciones relacionadas con el tema correspondiente. Así se hizo en la última de las citadas con investigadores del Jardín Botánico.

Habida cuenta del éxito de visitantes que cosechó la primera en los escasos día de su exhibición y las elogiosas críticas recibidas, consideramos la conveniencia de dar a conocer esta experiencia y lo hicimos con una Comunicación presentada al IV Coloquio gallego de Museos, celebrado en Pontevedra en 1994 (González y Rubio, 1994). De igual modo, procedimos con la que se ocupo de los instrumentos musicales con orra comunicación, al IV Congreso de la Sociedad Española de Musicología de 1997, en su Sección de Etnomusicología (Rubio, Baena y González, 1997).

Se ha tratado de experiencias sumamente positivas que han abierto nuevas posibilidades al trabajo de los alumnos universitarios, pero también a la colaboración entre determinados Departamentos y Museos como el citado, por no hablar de la establecida entre las tres disciplinas señaladas, clave en un planteamiento etnoarqueologico.

Con posterioridad, durante el año escolar 1999-2000, elegí como tema de Curso de Doctorado La reinterpretación de las primeras sociedades agricolas a traves de la Etnoarqueologia. En el mismo, hubo ocasión de revisar aquellas cuestiones que habian sido estudiadas desde estos planteamientos en el contexto citado y también sugerir otras posibilidades. Se analizaba, yendo de lo general a lo particular, el papel jugado por los datos provenientes de sociedades actuales en las teorías explicativas más recientes sobre la aparición de la agricultura y la adopción de la economía de producción. Se contemplaba como sus asentamientos suponían una aprovechamiento y una ordenación distintas del territorio, considerando que sedentarismo y almacenamiento constituian dos aspectos a debatir en relación con ellos. Los modos de adquisición del alimento, fundamentalmente las diversas formas de práctica agrícola y pastoril, y su repercusión en la organización del trabajo era otro de los temas tratados, así como el nuevo utillaje destinado a tareas también nuevas. Igualmente, se abordaban los estudios etnoarqueologicos relacionados con otros elementos del equipo material como, por ejemplo, la cerámica. Finalmente, las distintas for mas de organización social, los sistemas de intercambio y el mundo simbólico completaban la visión de los grupos neolíticos a través de la Etnoarqueología.

No obstante, como ya se ha puesto de manifiesto, son muchos los temas que quedan pendientes de interpretación a través de esta óptica. Precisamente, desearía proponer uno de ellos como colofón de este artículo por considerar que los datos etnográficos pueden sugerir interpretaciones alternativas o corroborar algunas de las hipótesis planteadas a nivel teórico. 


\section{PROPUESTA DE UN CASO DE ESTUDIO: RELACIONES ENTRE CAZADORES-RECOLEC- TORES Y AGRICUETORES}

La visión de las sociedades de cazadores-recolectores generada a partir de coloquios y estudios suficientemente conocidos (Lee y de Vore, 1968, en primer lugar; Sahlins, 1972; Schrire, 1984 e Ingold, Riches y Woodburn, 1988, entre otros) ha permitido, en una aparente paradoja, proporcionar también otra distinta de los procesos que provocan la existencia de los primeros grupos agrícolas. En nuestro país, autores como J. Mercader (19891990), por ejemplo, han planteado el paso de la caza-recolección a los inicios de la producción de alimentos en esa línea. La lista bibliográfica a este respecto sería extensísima en la actualidad, incluyendo obras de autores nacionales y extranjeros.

El autor citado señalaba en su momento (Mercader, 1989-1990): "Concebimos el origen de las estrategias de producción no como un gran cambio, sino como una variación del sistema cazador. Ésta puede entenderse únicamente si se contemplan la gran flexibilidad y multiplicidad de las estrategias económicas practicadas por las sociedades de cazadores-recolectores contemporáneos" Según eso, los verdaderos autores de la Revolución Neolítica (permítaseme utilizar un lenguaje tradicional) serían los cazadores-recolectores precedentes en cuyo contexto se operarian los cambios observables en la documentación arqueologica de los yacimientos clasificados como neolíticos.

Pero habría que recordar, de igual manera, que algunos de esos incipientes agricultores prehistóricos quienes quiera que fuesen, han sido los primeros de que tenemos noticia en la Historia a diferencia de los actuales que tienen tras de sí la experiencia acumulada (aunque quizá no individualmente) de milenios y vienen coexistiendo con sociedades de diverso tipo.

Globalmente hablando, en todo caso, cabría admitir que existe un punto de inflexión en diversas regiones del mundo en distintos momentos de la Prehistoria a partir del cual la sociedad agrícola se generaliza, lo que establecería también una diferencia con la situación de los cazadores-recolectores actuales que conviven con ella de forma habitual y. cuya distinción con los agricultores y horticultores no se percibe tan nítidamente en el contexto etnográfico. A este respecto, sería fundamental considerar qué es lo primordial en su economia, de qué modo marca su organización social y, sobre todo, qué se consideran ellos mismos.

Que las sociedades de cazadores-recolectores juegan un papel clave en la aparición de la economía de producción en la Prehistoria parece evidente. J.M. Vicent (1988, 56-58) había ya insistido en el hecho de que cuando el Neolítico se detecta arqueológicamente hablando, ciertos procesos se han operado ya (algunos como la domesticación aún no han concluido, en todo caso).

Podríamos decir que, incluso desde puntos de vista distintos, la literatura emanada de la Escuela Paleoeconómica de Cambridge así lo dejaba entrever, remontándose incluso al Paleolítico superior para buscar el origen de los mecanismos relacionados con la domesticación. El mismo J.G.D. Clark (1980, 7) indicaba que el Mesolítico "was an essential prelude to fundamental advances in the development of culture ${ }^{n}$ 
El proceso, en fin, no es lineal, eso es claro, yo misma lo he entendido de ese modo al estudiar el caso de la Península Ibérica (Rubio, 1981 y 1982), por lo que la coexistencia entre grupos de agricultores y de cazadores-recolectores ha debido ser un fenómeno corriente en las diversas áreas del mundo. En el caso de la Península Iberica y de otras zonas, los autores que han defendido posturas difusionistas desde diverso ángulo forzosamente lo han visto asi también (Rubio, 199751).

Pero, además de considerar la supuesta transición de una sociedad de caza y recolección a otra agrícola, cualquiera que sea la forma en que se produzca, cabría plantearse, lisa y llanamente, los términos de la citada relación en cualquier momento, dado que los cazadores-recolectores no se extinguen a pesar de la tendencia global antes aludida.

Sobre esta relación es sobre la que desearia proponer un caso de estudio que, a mi juicio, podría ayudar a su interpretación aportando sugerencias sobre aspectos poco o nada tenidos en cuenta. Obviamente, no pasa de ser lo que se indica: una propuesta y, por otra parte, es evidente que los datos etnográficos proceden de la bibliografia existente y no de un trabajo de campo propio.

En primer lugar, ¿qué nos dice la Prehistoria a este respecto?. Ya hemos visto cómo, desde planteamientos muy distintos se ha venido observando que la identificación de las sociedades neolíticas en la documentación arqueologica supone la consecución de un primer proceso operado en algunas sociedades de cazadores-recolectores, que se generaliza a nivel mundial, con excepciones. Dado que este proceso no es unilineal ni tampoco sincrónico, la coexistencia de distintos tipos de sociedades puede darse como cierta, hecho que persiste en la actualidad. ¿Cuâl es la diferencia fundamental?. Algunos grupos han sido los primeros absolutamente hablando que optan por este modo de adquisición del alimento, sin que quepa entrar aquí en los motivos que les impulsan a ello. A partir de ahi, las situaciones han sido múltiples y muy variadas.

¿Cabe pensar en que grupos incluso anteriores a los del Postglaciar pudieran haber poseído conocimientos suficientes sobre animales y plantas para desarrollar prácticas de cultivo y domesticación animal? Es posible, sin duda, pero también es cierto que este hecho no se ha detectado arqueológicamente y que es a partir de momentos cuya cronología conocemos cuando, en distintas regiones del mundo se produce la adopción progresiva y generalizada de dichas prácticas. A mi modo de ver, no habría que olvidar en estos procesos los de carácter biológico (transformación genotipica de animales y plantas) que, en la actualidad, o se soslayan o se estudian por separado. Por tanto, la cuestión podría plantearse a distintos niveles en momentos también diferentes.

Los cazadores-recolectores del Postglaciar, supuestamente organizados en bandas, habrían visto intensificarse las pautas relacionadas con el nacimiento de una cierta complejidad, iniciadas ya en el Paleolítico superior. M. Zvelebil (1986b, 7-8) resumía los dos modelos habitualmente establecidos para estas poblaciones: el de los llamados "cazadores-

[5] la bibliografia sobre el tema es tan amplia que rebasaría los límites de esze articulo, por lo que remito al serialado donde esta se recoge y analiza. 
recolectores complejos" ("affluent foragers") que, para A. Testart (1982), implicaría la presencia de almacenamiento a gran escala, y en segundo lugar, el de su consideración como un simple tránsito a la vida agrícola planteado desde diversos enfoques.

No obstante, en mi opinión y según lo dicho, las relaciones a que hago referencia se establecerían igualmente en un momento dado, con toda probabilidad, si elegimos el primer modelo, habida cuenta que finalmente la sociedad agrícola se impone a nivel global. Evidentemente, la excepción serían precisamente aquellas poblaciones que en la actualidad o en otros momentos de la Historia han mantenido una economía de caza y recolección, entre los que se cuentan precisamente aquellos cazadores-recolectores que consiguen desarrollar una mayor estabilidad. El segundo modelo genera el concepto de frontera agrícola con tres fases que, supuestamente, llevaron a la integración de las poblaciones del Postglaciar en el Neolítico o, dicho de otro modo, en la economía de producción (Zvelebil, 1986b, 12). Estas fases serían las ya conocidas: de disponibilidad, en la que se produciria un intercambio de materiales y de información entre cazadores-recolectores y agricultores, que continuarían su vida como unidades económicamente independientes; la de sustitución, en segundo lugar, que podría adoptar dos formas: externa (competencia entre cazadores-recolectores y agricultores por las tierras) e interna (los cazadores-recolectores adoptan otras estrategias de obtención del alimento) y, finalmente, la tercera, de consolidación, se percibe cuando existe una economía eminentemente neolítica (agricultura intensiva y extensiva).

En este sentido, la Europa templada ha proporcionado probables testimonios de dichos contactos. El propio M. Zvelebil (1986a), hacía alusión a la presencia de cerámica en grupos mesolíticos de la Europa nórdica que no alteraban su economía (cazadores de la foca anillada del sur de Suecia), pero que necesitaban recipientes para almacenar la grasa de estos animales destinada a combustible. En diversos yacimientos de la Europa nórdica y de la fachada atlántica, se ha insistido en este fenómeno: aparición de cerámica o de algunos animales domésticos en yacimientos de cazadores o de cazadores-pastores relativamente sedentarizados en zonas ricas en productos relacionados con el mar (van Berg, 1993). La aparición de los elementos citados se ha considerado el fruto de una circulación limitada de hombres, ideas y bienes, realizando cada uno dichos objetos con métodos propios. Ello explicaría la diversidad cerámica encontrada (van Berg, 1993, 414-415). Sin embargo, este hecho puede hacerse extensivo a otras áreas europeas y extraeuropeas. Una explicación alternativa sería que algunos de estos yacimientos fueran en realidad los campamentos de caza de los agricultores.

¿Qué datos nos proporciona la documentación etnográfica sobre los contactos a los que venimos aludiendo? Una de las escasas poblaciones actuales que mantienen, en cierto grado y en cierto modo, un género de vida de caza y recolección es la de los pigmeos africanos quienes, no obstante, mantienen relaciones muy estrechas con sus vecinos agricultores sin adoptar por ello su modo de vida en teoría superior o ventajoso según la opinión tradicional compartida por las autoridades políticas correspondientes, permaneciendo claramente diferenciados desde el punto de vista físico y cultural.

Los pigmeos presentan una apariencia diferente a la de sus vecinos negros bantúes. Tienen baja estatura (alrededor de 1'35 m.), cuerpo delgado y piernas cortas, piel pálida, 
pelo corto y frente alta y abultada. Su grupo sanguíneo los relaciona con los Khoisán (aborígenes de África meridional) (Turnbull, 1990, 114). Sin embargo, algunos de estos grupos presentan un color más oscuro a causa de la mezcla con sus vecinos cuya lengua hablan. En todo caso se trata de varios grupos étnicos distintos (Bahuchet, 1994, 38).

De los 150.000 pigmeos que sobreviven, unos 40.000 habitan en el bosque de Ituri al NE de la República Democrática del Congo (antiguo Zaire). Antes de la independencia (1960), la administración belga los había respetado, ya que vivian en una zona inaccesible y no aprovechable para el dominio colonial (Figura 1). Los relatos de los primeros viajeros los describían alternativamente como "nobles e igualitarios enanos de los bosques", o como salvajes feroces e ingobernables, restos de una fase anterior de la evolución humana (Bahuchet, 1994, 38).

Desde el punto de vista físico y social, están muy bien adaptados a la vida de la selva ecuatorial que, para ellos, resulta un lugar "cálido y agradable" (Turnbull, 1990, 115-116). Se hallan divididos en cuatro grupos que, a su vez, pueden subdividirse en otros. Son los Binga o Ba Binga que habitan en la selva occidental, al norte del río Congo y al este del río Ubangi; los Twa centrales; los Bagesera y los Mbuti (o Bambuti) que ocupan la selva ecuatorial del centro y este del continente, al este de Kisangani, cerca del extremo norte del río Congo. Los Binga y los Mbuti son denominados Twa o Tswa con un sentido peyorativo por los habitantes, no pigmeos, de los poblados vecinos. Pero, subgrupos de pigmeos pueden hallarse también en la región meridional de la República Centroafricana, centro y sudeste de Camenín, noreste de Gabón y regiones montañosas de Ruanda. Hablan lenguas distintas y pueden utilizar técnicas de caza peculiares.

Los Mbuti son los que, hasta cierto punto, han conservado su género de vida tradicional y sus características a pesar del contacto con vecinos más numerosos y tecnológicamente distintos, "superiores" según las categorías evolucionistas tradicionales. En la actualidad, puede decirse que vuelven a vivir en su primitivo territorio a pesar de que su vida se vió alterada por la llegada de los campesinos bantúes. Los Mbuti nunca contraen matrimonio con mujeres de los poblados y los hijos de sus mujeres, habidos con hombres de los mismos, son rechazados. Realmente, la vida de los pigmeos está marcada por la relación con estos vecinos con los que coexisten aunque sus economias sean contrarias: la de los pigmeos se basa en la conservación de la selva, la suya en la destrucción de la misma (Turnbull, 1990, 116).

Las características de los grupos pigmeos que conservan los modos de vida tradicionales son las propias de los cazadores-recolectores. La banda, por tanto, sería su modo de organización social. Pero el grupo se halla en un continuo cambio y la única definición posible sería la de un grupo de gente que caza en colaboración. Son agrupaciones flexibles que pueden admitir nuevos miembros o expulsar a otros si llega el caso. Por su movilidad resultan difíciles de controlar. La recolección es para ellos tan importante en su alimentación como la caza. Sin embargo, los Mbuti se ven a sí mismos fundamentalmente como cazadores. En el mundo selvâtico en el que habitan, los animales tienen tanta importancia como los hombres. Por ello, la leyenda señala que la necesidad de matar es debida a un pecado original. Así pues, como acto propiciatorio se enciende una hoguera antes de cada caceria. Como contrapartida, matar mâs de lo necesario es pecado, salvaguardando así 
a través de los principios religiosos la integridad de la selva. Sin embargo, como ésta ha de ser explotada para poder subsistir, los Mbuti creen que se halla dotada de una "bondad divina" especial que les permite continuar su forma de vida.

Las técnicas de caza poseen su propia organización: los grupos que cazan con redes habitan en la parte occidental y los que lo hacen con arco en la oriental. Los primeros se agrupan en 6-7 familias y los segundos en 3-4, implicando el primer sistema la participación de hombres, mujeres y niños. En todo caso, un grupo de cazadores nunca puede sobrepasar las 30 familias, ya que, en ese caso, la caza sería muy difícil además de improductiva.

Una vez al año, en la "estación de la miel", los pigmeos se dedican a la recogida de ésta y se apartan de la rutina anual. Los arqueros se dedican a la "begbe" o batida de caza, en la que se adoptan los métodos de cooperación de los cazadores con redes aunque sin ellas. En cambio, estos segundos se dividen en pequeños grupos que recogen frutos silvestres, bayas, setas y raíces, viviendo tan sólo de miel y de lo que consiguen cazar con arcos y flechas. En esa época, los arqueros se pueden reunir y actuar como una comunidad después de haber vivido aislados y los de redes, por su parte, liberarse de cualquier desavenencia antes de reanudar su técnica basada en la colaboración.

La solución más corriente ante un posible conflicto con otro miembro de la tribu es trasladarse de campamento con la excusa de visitar a un pariente, por ejemplo. No hay métodos formales de resolver los conflictos por lo que esta práctica es la común y, por otro lado, implica un contacto social más allá de los márgenes del propio territorio.

El grupo de cazadores constituye para el individuo una auténtica familia, más unida a él que sus propios parientes. No hay una autoridad central ni generación dominante ni "big-man", pero cada grupo de edad tiene sus propias responsabilidades y funciones: los niños encienden la hoguera propiciatoria; los jóvenes son los portavoces del grupo, así como sus jueces y en la ceremonia matinal ("molimo madé") hacen sonar la trompeta ritual ("molimo") que representa el espíritu de la selva (si éste se manifiesta a través de los jóvenes para expresar su disgusto, lo hace atacando las chozas de los que han transgredido la ley); los adultos son responsables de la economía y son severamente criticados por los ancianos; finalmente, éstos son los átbitros de la sociedad y controlan el "molimo magbo" (festival que se celebra para conmemorar a los muertos y aplacar a los dioses). La trompeta se utiliza en este caso para entrar en comunicación con la divinidad de la selva que resolverá los problemas del grupo (Turnbull, 1990, 116-120).

Para los Mbuti, los cantos, sobre todo, y las danzas se ejecutan para hacer felices a los espíritus. En general, los pigmeos destacan por su canto polifónico (Bahuchet, 1994, 39). Este, el canto, les complace especialmente porque es "frío como la selva umbrosa y además requiere el aliento, la fuerza vital y el esfuerzo" El único canto con motivación individual son las nanas que cantan las madres a sus hijos, el resto son comunales y los considerados legítimos fundamentalmente. Hay cantos relacionados con danzas alusivas a la caza en las que se imitan los movimientos del animal o del cazador; otros se relacionan con la búsqueda de la miel (el hombre simula ser el recolector y la mujer la abeja que ataca e intenta picar); algunos más, finalmente, se relacionan con ritos de paso (Kaemmer, 1993, 
30 y 40-41). Pero veremos cómo también este aspecto se halla claramente diferenciado en las relaciones con sus vecinos.

En una época difícil de precisar, quizá en el inicio de nuestra era, giupos de bantúes, término que hace referencia, sobre todo, a características lingüisticas comunes, emigraron desde las orillas del río Benné en África occidental hacia las selvas de la cuenca del río Congo. Una parte se estableció en sus orillas y otros continuaron su marcha a través de las selvas en siglos posteriores. En los siglos VIII y IX, comunidades bantúes comerciaban con cobre en las cosłas de África oriental. Con posterioridad, se dispersaron en distintas direcciones: hacia el sur, el este, de nuevo hacia las selvas del Congo, al NE y NW Aquí se detuvo el avance por la migración hacia el sur de los habitantes del Nilo. Estas poblaciones bantúes poseían el conocimiento del hierro desde antes del siglo III a.C. en África occidental y de la agricultura con fecha anterior aún. Antes de la llegada de los europeos (siglo XV) poseían diversos tipos de organización social. A su llegada a la cuenca del Congo, los pigmeos y bosquimanos vivian en la Edad de Piedra, según señala V.G.K. Pons (1990, 112113).

Una vez instaladas las poblaciones bantúes, es preciso considerar como una referencia las relaciones establecidas entre los grupos de agricultores y los de cazadores-recolectores preexistentes, los pigmeos en este caso concreto.

Estas relaciones podrian calificarse de simbióticas y de beneficiosas para ambos. Los pigmeos tienen como hábitat natural la selva tropical y los agricultores se instalan en los límites de la misma que para ellos resulta un lugar prohibido. Cada uno proporciona al otro servicios esenciales, manteniendo separada su identidad cultural. Sin embargo, los agricultores consideran a sus vecinos pigmeos como sus "esclavos", compadeciéndoles por vivir en la selva. A cambio, éstos se burlan de ellos por el miedo que experimentan hacia los espiritus de la misma.

Durante generaciones, los pigmeos del Congo han intercambiado con sus vecinos miel y carne por frutos cosechados (plátanos y names)(Kottak, 1994, 156). El intercambio que practican es un ejemplo de reciprocidad negativa (se trata de ganar más que el compañero de transacción) y podría calificarse de "silencioso", ya que durante el mismo no hay contacto personal. Un mbuti deja los productos en un lugar acostumbrado. Los horticultores los recogen y dejan los suyos a cambio. Si una parte piensa que el retorno es insuficiente, lo deja en el lugar de costumbre sin recogerlo y, si la otra parte quiere continuar con el intercambio, lo incrementará (Kottak, 1994, 176).

En los rituales, los pigmeos dan la impresión de comportarse como subordinados de los agricultores y de depender de ellos por lo que respecta a la comida, al vestido y a las ceremonias, pero realmente consiguen su meta recluyéndolos en el poblado. A su vez, los bantúes, tienen la sensación de controlar a los pigmeos.

En la ceremonia de iniciación, "nkumbi", uno de los ritos más importantes, participan tanto unos como otros, sirviendo para definirlos como personas, para separar el mundo del poblado del de la selva e incluso diferenciar el bien del mal (Turnbull, 1990, 117-118). Hay cantos y danzas bantúes que se interpretan exclusivamente en el poblado, pero nunca en la selva (Kaemmer, 1993, 40-41). 
El hierro se ha convertido en imprescindible para los agricultores de los poblados. Con êl fabrican cuchillos, hojas de hachas y azuelas. Sin embargo, para el cazador no es esencial: las lanzas con la punta endurecida a fuego resultan tan efectivas como las de metal (Tumbull, 1990, 116-120).

Un segundo tipo de relaciones que es preciso comentar se produce con los distintos gobiernos del estado nacido con posterioridad a la independencia. Los intentos de éstos y de los misioneros de "liberar" a los pigmeos de la selva de la esclavitud a la que se someten y son sometidos por sus vecinos no han tenido éxito, dado que se hallan habituados a su medio natural. Se hicieron claros en la selva y se construyeron asentamientos pigmeos. Al comienzo, estos grupos reaccionaron con entusiasmo ante los útiles granitos, las semillas para sembrar, las escuelas y los dispensarios. Posteriormente, sin embargo, volvieron a la selva, consumieron el grano y vendieron los útiles a sus vecinos. No tenía sentido trabajar la tierra cuando se podía intercambiar o robar a los agricultores. Por otra parte, los más mayores ofrecían una fuerte resistencia a cualquier cambio.

Otro tipo de problemas se ha añadido a los ya citados. Fuera de la selva estas gentes no pueden sobrevivir. Contraen enfermedades que antes no tenian, incluso el agua que beben, inofensiva para los agricultores negros vecinos, es nociva para ellos. En uno de los poblados modelo, murieron veintinueve personas en un solo día a causa de la exposición al sol (Brain, 1976, 212-214).

Con todo, la situación es diversa para otros grupos de pigmeos que han comenzado a cultivar y a adquirir útiles de hierro y cerámica de los agricultores (Bahuchet, 1994, 39). Por otra parte, algunos de los que han abandonado la selva y su modo de vida han pasado a constituir una atracción turística y son despreciados por el resto. En una o dos generaciones, adquieren los caracteres físicos, los valores y los patrones de comportamiento de los grupos negros vecinos, con lo que su supervivencia resulta más que problemática (Turnbull, 1990, 116-120).

Si aplicamos la simple analogía, una serie de rasgos nos sugieren determinadas similitudes con grupos prehistóricos. Sin embargo, las diferencias se evidencian igualmente y son éstas las que realmente pueden sugerir explicaciones alternativas a las existentes. El primero de ellos haría referencia a los contactos entre grupos de cazadores-recolectores y colonos (literalmente aquî) agrícolas. En este caso, además, ambas poblaciones son distintas incluso físicamente, algo que podría interesar en planteamientos próximos a los estudios de genética de las poblaciones relacionados con el avance de la economía de producción sobradamente conocidos. Sin embargo, lo que aquí se produce es un doble proceso: el mantenimiento intencionado de las diferencias, por un lado, y una rapidísima absorción cuando éste no se da. Por no hablar de las diferencias de un equipo material que, en este caso concreto, incluye la metalurgia (elemento éste que desvirtuaría el paralelismo con grupos neolíticos). Sin embargo, cabría también plantear cuál ha sido la repercusión real de ésta en la economía de los grupos de agricultores bantúes.

Pero la perspectiva histórica es necesaria. No han sido iguales los contactos establecidos con los bantúes llegados en primera instancia, a partir del inicio de nuestra era, que 
con los actuales. Ni los de ambos con la administración belga primero y con la surgida con posterioridad a la independencia.

Precisamente, el carácter de estos contactos reforzaría lo expuesto en aquellas teorias que explican la aparición de la agricultura (y por ende de la economía de producción), solamente cuando los cazadores-recolectores se ven forzados a adoptarla por el motivo que sea (Cohen, 1981, por ejemplo). Pero aquí, algunas de las causas habitualmente propuestas no parecen darse y otras no se revelan como demasiado convincentes para los mismos. La presión demográfica y el desequilibrio entre recursos y población obviamente no se produce, ya que los grupos de pigmeos tienden a desaparecer por integración o por causas más dramáticas. Ni siquiera los esfuerzos del gobierno del Congo en este sentido han tenido éxito. La visión de la vida agrícola como algo ventajoso y superior aparece de nuevo como un tópico, no siempre válido. Ni siquiera la liberación de uná supuesta esclavitud tiene aquí ningún peso. Lo mismo sucede con un utillaje más sofisticado tecnológicamente hablando que para el cazador pigmeo no presenta ninguna utilidad.

Se trata de un caso de adaptación extremo que nos lleva a recordar, estableciendo las oportunas diferencias, grupos (culturas del Postglaciar) en los que ésta parece ser la palabra clave para explicar satisfactoriamente sus modos de vida o planteamientos en los que la cultura se contempla como un sistema adaptativo (Binford, 1968). Dicha adaptación marca las actividades, el mundo simbólico e incluso la propia integridad física del individuo.

La organización social de los pigmeos, cazadores según su propia definición, encaja claramente como cabría esperar en la de bandas, con procedimientos encaminados a la eliminación de conflictos y a proporcionar cohesión a grupos extremadamente móviles. Estos mecanismos están relacionados tanto con las actividades económicas como con el mundo de las creencias (cambios de actividad y organización estacional que, de alguna manera, implicarían que unos ocuparan temporalmente el lugar de los otros, asi como rituales).

Las creencias, la ideología en general, nos sugieren un buen número de posibilidades y representan posiblemente los aspectos más interesantes de un esrudio como el propuesto.

De entrada, la relación establecida es satisfactoria para ambas sociedades. Simbiosis sería el término más adecuado para ella. Sin embargo, la reciprocidad negativa nos induciría a pensar que, estableciéndose el intercambio entre grupos desiguales, el más dotado tecnológicamente hablando e ideológicamente dominante como en apariencia serian los bantúes, llevaría la mejor parte. No está claro que esto ocurra así en el caso a que nos referimos. Pero hasta tal punto se produce una situación cómoda, que los pigmeos que adquieren o roban (o aceptan gratuitamente) de sus compañeros de intercambio o de autoridades políticas diversos elementos y objetos no optan por la producción de los mismos. A su vez, nadie les niega estos bienes ni les obliga realmente a procurárselos por su propio esfuerzo, ya que los agricultores vecinos tienen solucionadas a través de ellos otras necesidades y los gobiernos no han estado, de momento, interesados en ocupar este territorio. Tal situación, sin embargo, puede cambiar de un día para otro. 
La supuesta situación de esclavitud que sufren los pigmeos parece constituir únicamente una coartada para ellos y para los agricultores, lo mismo que su actitud despectiva, indudablemente recíproca por parte de los pigmeos. Pero queda claro que para éstos no tiene nada de desventajoso, al contrario, contribuye a mantener a cada uno en su lugar El control de los bantúes sobre los pigmeos es igualmente ilusorio: la propia movilidad de estos segundos lo hace prácticamente imposible y el miedo a la selva de los primeros refuerza esta situación. Los pigmeos además tienen perfectamente clara su identidad como hemos visto: cazadores cuya actividad depredadora se justifica también por creencias religiosas.

Llegamos pues al punto clave de la relación: la frontera social e ideológica que se perfila y se mantiene a base de creencias y actitudes expresadas en los rituales señalados: supuesto dominio de los bantúes (y servidumbre de los pigmeos); celebración conjunta de rituales que sirven, paradójicamente, para señalar el lugar de cada uno; tabúes religiosos que mantienen a cada uno en el medio ambiente al que se hallan adaptados lo mismo que cantos y danzas destinados a ser interpretados en el lugar de cada ano pero no en el del vecino e, incluso, extrema rigidez de las normas sobre las relaciones mantenidas por pigmeos y bantúes.

De nuevo, se refuerza aquí el rasgo a menudo destacado en las teorías antes aludidas que tratan de explicar el nacimiento de la agricultura por causas de fuerza mayor y no por las "evidentes" ventajas de la misma, como es el carácter "conservador" de los cazadores-recolectores. No sólo habría aquí resistencia a adoptar la agricultura, sino también la metalurgia, imprescindible en cambio para el utillaje de los agricultores. Los más mayores, en este caso, son absolutamente reacios a cualquier cambio. La rápida absorción, incluso física, de los que abandonaron el modo de vida tradicional puede motivar también dicha actitud, así como el desprecio ante el papel de simple atracción turística al que algunos han sido relegados.

Por lo tanto, el concepto de frontera ideológica habrá también de ser considerado en las relaciones entre cazadores-recolectores y agricultores, ya que, de hecho, aquí al menos parece ser aún más fuerte que otros planteamientos e incluso que invitaciones externas. Desde este punto de vista, las fases establecidas por $\mathrm{M}$. Zvelebil para el Postglaciar europeo, por ejemplo, que fundamentalmente contemplan procesos económicos, resultarían excesivamente rígidas y lineales, ya que esta secuencia podría quedar interrumpida indefinidamente en algún punto por causas como las vistas aquí.

Sin duda, nos hallamos ante un caso que reúne condiciones muy concretas: baja densidad demográfica, adaptación completa al medio ambiente y facilidad de aprovisionamiento de ciertos productos, por lo que cabría plantearse qué posibilidades de generalización existen a partir del mismo. Dada la situación observada en el contexto etnográfico y también en el arqueológico no parece infundado proponer la existencia de situaciones similares durante la Prehistoria. Desde luego, si nos atenemos al propio continente africano, donde se produciría la más pura continuidad histórica, la adopción de la agricultura en la mitad subsahariana es muy tardía y lenta e, incluso como hemos visto, no siempre se produce. Posiblemente, también en otras zonas del mundo podría pensarse en fronteras similares. 
Sugerencias de esta indole enriquecen, en mi opinion, las posibilidades de interpretación de ciertos yacimientos a menudo catalogados desde una perspectiva excesivamente lineal, pero que muestran la variabilidad de la documentación arqueológica existente en determinados horizontes neolíticos, por ejemplo.

Una vez más, la recogica de la documentación que estas poblaciones aportan es fundamental, ya que su funuro es poco halagüeño, entre otras cosas, porque cuando se vence la resistencia que ofrecen, su integración es rapidísima, como demuestran los grupos que han dejado la selva y que supondrian la otra cara de la moneda: aquellos para los que el abandono de su modo de vida ha supuesto una ventaja.

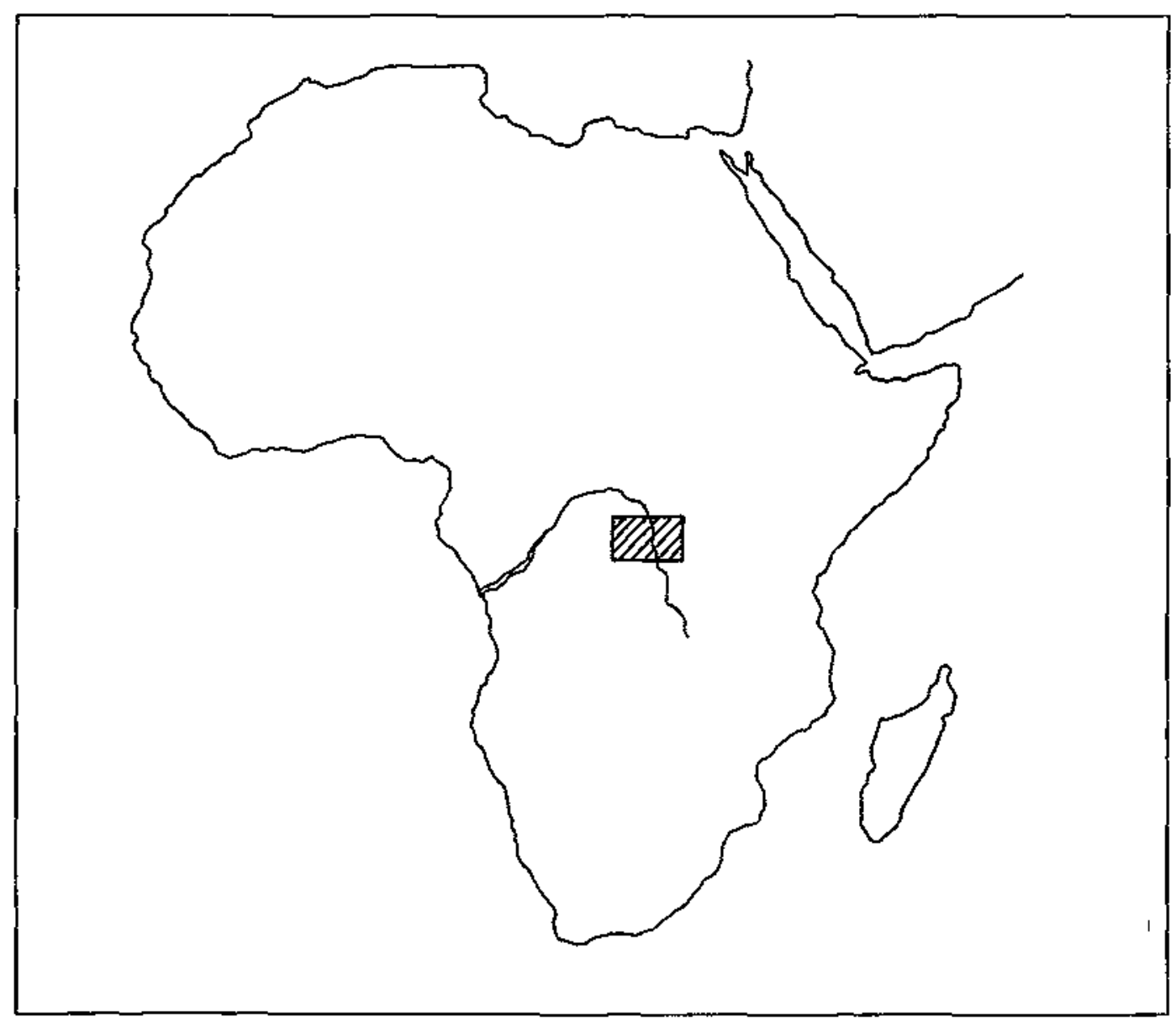

Figura 1. Sizuación aproximada del grapo pigneo Mbuti en ia actualidad (a partir de Burenhult 1994, 10, 18). 


\section{BIBLIOGRAFIA}

ALMAGRO, M. 1967: Introducción al estudio de la Prebistoria y de la Arqueologia de campo, Madrid.

ASHER, R. 1961: "Analogy in Archaeological Interpretation", Southwestern Journal of Anthropology, 17, 317-325.

AURENCHE, O. (Dir.) 1984: Nomades et sédentaires. Perspectives etbnoarchéologiques, París.

- 1995: "Les conditions de l'enquête ethnoarchéologique", BAZZANA, A. y DELAIGUE, M.C. (Êds.): Etbno-arcbéologie méditerranéenne, Madrid, 13-16.

BAHUCHET, S. 1994: Los pigmeos de Africa central, BURENHULT, G. (Ed.) 1993-1994: Atlas culturales de la Humanidad, Madrid, 10: Continuidad y cambio, 38-39.

BAZZANA, A. y DELAIGUE, M.C. (Éds.) 1995: Etbno-arcbéologie méditerranéenne, Madrid.

BERG, P.L. van 1993: "Ceramiques de chasseurs et ceramiques d'agriculteurs en Europe" Actes du XIle Congrès International des Sciences Prébistoriques et Protobistoriques (Bratislava, 1-7, septembre), 2, 413-415.

BINFORD, L.R. 1962: "Archaeology as Anthropology", American Antiquity, 28.2, 217-225.

- 1968: Post-Pleistocene Adaptations, BINFORD, L.R, y BINFORD, S.R. (Eds.): New Perspectives in Arcbaeology, Chicago.

- 1988: En busca del pasado, Barcelona.

BRAIN, R. 1976: The last primitite people, Nueva York.

CERRILLO, E. 1989: "Etnoarqueología de la vida rural", Antropología cultural en Extremadura. Primeras jornadas de cultura popular, Mérida, 541-555.

CLARK, J.G.D. 1980: Mesolithic prelude, Edinburgh.

COHEN, M.N. 1981: La crisis alimentaria en La prebistoria, Madrid.

CRIBB, R. 1991: Nomads in arcbaeology, Cambridge.

FERNÁNDEZ, V.M. 1994: "Emoarqueología: una guía de métodos y aplicaciones", Retista de Dialectología y Tradiciones Populares, XLIX, 137-169.

FERNÁNDEZ, M. et al. 1994: "Aproximación al estudio etnoarqueológico del Guadiana menor (Jaén)", Trabajos de Prebistoria, 51 1, 111-125.

FRIED, M. 1967: The evolution of political society, Nueva York.

FRIEDMAN, J. y ROWLANDS, M.Q. (Eds.) 1978: The evolution of social systems, Londres.

GODELIER, M. 1971: Esquemas de evolución de las sociedades, Madrid.

GONZÁLEZ, C. y RUBIO, I. 1994: "La investigación etnoarqueológica: una experiencia a través del Departamento de Prehistoria y Arqueología y del Museo de Artes y Tradiciones Populares de la Universidad Autónoma de Madrid" Actas del $I V$ Coloquio galego de Museos. Investigación y Museos (Pontevedra, 14-16 de diciembre de 1994), 93-99.

-, - y VALIENTE 1995: "Arquirectura popular en Madrid. Algunas construcciones auxiliares de los términos de Valdilecha, Campo Real, Olmeda de las Fuentes, Villar del Olmo, Corpa y Nuevo Baztán", Etnografía Española, 9, 49-97

GOULD, R. (Ed.) 1978: Explorations in Etbnoarcbaeology, Albuquerque.

- 1980: Liting Arcbaeology, Cambridge. 
GUIDI, A. 1988: Storia della Paletnologia, Roma

HARRIS, M. 1978: El desarrollo de la teoria antropológica, Madrid.

HERNANDO, A. 1995: "La Etnoarqueologia, hoy una vía eficaz de aproximación al pasado", Trabajos de Prebistoria, 52.2, 15-30.

HODDER, I. 1988: Interpretación en Arqueología. Corrientes actuales, Barcelona.

INGOLD, T 1985: "The significance of storage in hunting societies", GAST, M., SIGAUT, F. Y BEUTLER, C. (Eds.): Les tecbniques de conservation des grains à long terme, París, 553-571

- RICHES, D. Y WOODBURN, J. (Eds.) 1988: Hunters and gatberers, Oxford.

KAEMMER, J.E. 1993: Music in Human Life, Austin.

KOTTAK, C.P. 1994: Antropologia, Madrid.

KRAMER, C. (Ed). 1979: Etbnoarchaeology, Nueva York.

LAURENCICH-MINELII, L. 1996: "Cenni sulla storia dell'Etnoarcheología americanista", Bollettino del XIII Congresso dell'Unione Internazionale delle Scienze Preistoriche e Protostoricbe, Forlà, 3.

LEE, R. y DE VORE, I. (Eds.) 1968: Man the bunter, Chicago.

- y (Eds.) 1976: Kalabari bunters and gatberers, Cambridge, Mass.

MERCADER, J. 1989-1990: "Nuevas perspectivas sobre el final de la caza-recolección y los inicios de la agricultura/ganaderia", Kalathos, 9-10, 47-64.

-1992: "Cazadores-recolectores y antropólogos del sigio XXI", Revista de Arqueología, 140, $40-50$.

- 1993: "Nuestros vecinos cazadores-recolectores al borde del siglo XXI: revisionismo y etnoarqueología en los estudios de caza-recoleccion", Antropología, 4-5, 183-199.

MERCIER, J. 1995: Historia de la Antropología, Barcelona.

MOUINOS, M. et al. 1996: "Arqueología para después de una feria", Arqueología espacial, 15, 149-183.

MUNTION, C. y VEGAS, J.I. 1991. "Una excavación arqueoígica en el corazón de la trashumancia riojana", VICENTE, E. y GRANDE, J (Coords.): Sobre cultura pastorll. Madrid, 255-281.

ORME, B. 1973: "Archaeology and ethnography", RENFREW, C. (Ed.): The explanation of cultural change, Londres, $481-492$.

- 1981: Antbropology for arcbaeologists: An Introduction, Washington.

PETREQUIN, A.M. y PETREQUIN, P. 1988: Le Néolithique des lacs, París.

PONS, V.G.K. 1990: Los pobladores de la Cuenca del Congo, EVANS-PRITCHARD, E. (Dir.) 1990: Pueblos de la tierra, Barcelona, Africa tropical, 112-113.

RATHIE, W.L. 1974: "The Garbage Project: a new way of looking at the problem of Archaeology", Arcbaeology, 27.4, 236-241.

RENFREW, C. Y BAHN, P. 1993: Arqueología. Teorias, mêtodos y práctica, Madrid.

RUBIO, I. 1981: Aspectos socioeconómicos del Neolitico peninsular, Tesis doctoral inédita, U.A.M.

-1982: "La economía de la Península Ibérica durante el Neolítico. Sus inicios", Le Nêolitbique ancien méditerranéen. Actes du Colloque International de Prébistoire (Montpellier 1981), 181-190. 
- 1997: "El paradigma difusionista y la neolitización de la Península Ibérica: una explicación recurrente", CuPAUAM, 24, 9-58.

-BAENA, J, y GONZÁLEZ, C. 1997: "Etnoarqueología y música: flautas y silbatos primitivos", Actas del IV Congreso de la Sociedad Española de Musicología (Madrid, 8-10 de majo), Revista de Musicologia, XX.2, 867-874.

SAHLINS, M. 1972a: Las sociedades tribales, Barcelona.

- 1972b: Stone Age economics, Chicago.(1977: Economía de la Edad de Piedra, Madrid).

SCHIFFER, M.B. 1976: Bebavioural arcbaeology, Nueva York.

SCHRURE, C. 1984: Past and present in bunter-gatberer studies, Orlando.

SERVICE, E. 1971: Primitive Social Organization, Nueva York.

- 1973: Los cazadores, Barcelona.

- 1984: Los origenes del Estado y de la civilización, Madrid.

STILES, D. 1977: "Ethnoarchaeology: a discussion of methods and applications", Man, 12, 86-99.

TESTART, A. 1982: "The significance of food storage among hunter-gatherers: residence, patterns, population densities, and social inequalities", Current Antbropology, 23, 523-537.

- 1988: "Some Majors Problems in the Social Anthropology of Hunter-Gatherers", Current Antbropology, 29.1, 1-31.

TRIGGER, B.G. 1992: Historia del pensamiento arqueológico, Barcelona.

TUNRBULL, C. 1990: Los Pigmeos-Cuenca del Congo, EVANS-PRITCHARD, E. (Dir.) 1990: Pueblos de la tierra, Barcelona, Africa tropical, 114-120.

VICENT, J.M. 1988: "El hombre cazador se hace agricultor", Historia 16, 142, 56-67

VOSSEN, R. 1990: "Analogies in Ethnoarchaeology: Pitfalls and Possibilities", SUGIURA, Y. y

SERRA, C.: Etnoarqueología. Primer coloquio Boscb-Gimpera, México, 23-41

V.AA. 1995: Una visión etnoarqueologica de la tecnología cerámica, U.A.M.

- 1995-1996: La caza: instrumentos y estrategias, U.A.M.-Córdoba.

- 1997: Los instrumentos musicales en la Prehistoria y en las sociedades tradicionales actuales, U.A.M.-Aranda de Duero (Burbos).

- 1998: El uso de fibras vegetales en las sociedades prebistóricas y tradicionales, U.A.M.

WOODBURN, J. 1980: "Hunters and Gatherers today and reconstruction of the Past", GELLNER,

E. (Ed.): Soviet and Western Antbropology, Londres.

YELLEN, J.E. 1977: Arcbaeological approaches to the present. Models for reconstructing the past, Nueva York.

ZVELEBIL, M. 1986a: "Busca del alimento en los bosques de la Europa postglacial", Investigactón y Ciencia.

-1986b: Mesolitbic prelude and neolitbic revolution, ZVELEBIL, $M$. (Ed.): Hunters in transition, Cambridge, 5-15. 